\title{
BMJ Open Rationale and design of a randomised, double-blind, placebo-controlled, parallel-group, investigator-initiated phase 2a study to investigate the efficacy and safety of elobixibat in combination with cholestyramine for non-alcoholic fatty liver disease
}

\author{
Takaomi Kessoku (D) , , ${ }^{1,2}$ Takashi Kobayashi, ${ }^{1}$ Anna Ozaki, ${ }^{1}$ Michihiro Iwaki, ${ }^{1}$ \\ Yasushi Honda, ${ }^{1,2}$ Yuji Ogawa, ${ }^{1}$ Kento Imajo, ${ }^{1}$ Yusuke Saigusa, ${ }^{3}$ Koji Yamamoto, ${ }^{3}$ \\ Takeharu Yamanaka, ${ }^{3}$ Haruki Usuda, ${ }^{4}$ Koichiro Wada, ${ }^{4}$ Masato Yoneda, ${ }^{1}$ \\ Satoru Saito, ${ }^{1}$ Atsushi Nakajima (i) ${ }^{1}$
}

To cite: Kessoku T, Kobayashi T, Ozaki A, et al. Rationale and design of a randomised, double-blind, placebo-controlled, parallelgroup, investigator-initiated phase 2a study to investigate the efficacy and safety of elobixibat in combination with cholestyramine for non-alcoholic fatty liver disease. BMJ Open 2020;10:e037961. doi:10.1136/ bmjopen-2020-037961

- Prepublication history and additional material for this paper are available online. To view these files, please visit the journal online (http://dx.doi. org/10.1136/bmjopen-2020037961).

Received 22 February 2020 Revised 01 July 2020 Accepted 08 July 2020

Check for updates

(C) Author(s) (or their employer(s)) 2020. Re-use permitted under CC BY-NC. No commercial re-use. See rights and permissions. Published by BMJ.

For numbered affiliations see end of article.

Correspondence to Professor Atsushi Nakajima; nakajima-tky@umin.ac.jp

\section{ABSTRACT}

Introduction Non-alcoholic fatty liver disease (NAFLD) pathogenesis involves abnormal metabolism of cholesterol and hepatic accumulation of toxic free-cholesterol. Elobixibat (EXB) inhibits the ileal bile acid (BA) transporter. EXB and cholestyramine (CTM) facilitate the removal of free cholesterol from the liver by decreasing $B A$ recirculation to the liver, thereby stimulating novel BA synthesis from cholesterol. In this randomised, doubleblind, placebo-controlled, parallel-group, phase lla study, we aim to provide a proof-of-concept assessment by evaluating the efficacy and safety of EXB in combination with CTM in patients with NAFLD.

Methods and analysis A total of 100 adult patients with NAFLD, diagnosed based on low-density lipoprotein cholesterol (LDL-C) level of $>120 \mathrm{mg} / \mathrm{dL}$ and liver fat content of $\geq 8 \%$ by MRI-based proton density fat fraction (MRI-PDFF), who meet the inclusion/exclusion criteria will be enrolled. The patients will be randomly assigned to receive the combination therapy of $10 \mathrm{mg}$ EXB and $9 \mathrm{~g}$ CTM powder (4 g CTM), 10 mg EXB monotherapy, $9 \mathrm{~g}$ CTM powder monotherapy or a placebo treatment $(n=25$ per group). Blood tests and MRls will be performed 16 weeks following treatment initiation. The primary study endpoint will be the absolute LDL-C level change at week 16 after treatment initiation. The exploratory endpoint will include absolute changes in the liver fat fraction as measured by MRI-PDFF. This proof-of-concept study will determine whether the combination therapy of EXB and CTM is effective and safe for patients with NAFLD.

Ethics and dissemination Ethics approval was obtained from the Ethics Committee of Yokohama City University Hospital before participant enrolment. The results of this study will be submitted for publication in international peer-reviewed journals and the key findings will be presented at international scientific conferences.

\section{Strengths and limitations of this study}

- This study is the first randomised, double-blind, placebo-controlled, phase 2a trial to determine the efficacy and safety of elobixibat in combination with cholestyramine for nonalcoholic fatty liver disease.

- This original proof-of-concept study aims to explore the additive effects and the reduction in the adverse events following combination therapy, compared with those of monotherapy.

- The primary outcome is the improvement in lowdensity lipoprotein cholesterol, and the exploratory outcome is liver fat content assessed by magnetic resonance imaging-proton density fat fraction.

- Limitations are short treatment duration and the lack of liver biopsy.

Trial registration number NCT04235205

\section{INTRODUCTION}

Non-alcoholic fatty liver disease (NAFLD) is a clinical condition that is detected by tissue or image analyses and diagnosed by excluding alcoholism and other liver diseases. It is the hepatic manifestation of a metabolic syndrome and is often associated with obesity, diabetes mellitus, dyslipidaemia, hypertension and other disorders. The prevalence of NAFLD is increasing worldwide; in Japan, it increased from $12.9 \%$ in 1994 to approximately $34.7 \%$ in $2000 .{ }^{1}$ NAFLD is classified as non-alcoholic fatty liver (NAFL) and nonalcoholic steatohepatitis (NASH), which 
includes inflammation and a progressive disease associated with liver cancer or cirrhosis, affecting $10 \%-20 \%$ of the patients. ${ }^{2}$ The factors contributing to NASH pathogenesis are abnormal cholesterol metabolism and free cholesterol accumulation in the liver, which is toxic to hepatocytes, leads to inflammation and fibrosis. ${ }^{3}$

Bile acids (BAs) may be critical in NASH pathogenesis. ${ }^{4-6}$ They act as signalling molecules in lipid, glucose and energy homeostasis, whose metabolic pathways are linked to NAFLD/NASH and comorbidities, including metabolic syndrome, obesity and diabetes. The BAs and free cholesterol, as the precursor for BA synthesis, can act as lipotoxic agents that drive inflammation and fibrosis. In previous studies, both serum and liver BA levels were elevated in patients with NASH, and recent data suggested that the occurrence and severity of NASH are associated with specific changes in circulating BAs. ${ }^{6}$ Therefore, targeting the BA pathways may have a therapeutic potential for patients with NAFLD.

Elobixibat (EXB) inhibits the ileal BA transporter (IBAT) and is approved for marketing as an oral treatment for chronic constipation. ${ }^{7}$ The IBAT, expressed primarily in the distal ileum, is a key element in the enterohepatic circulation of BAs, as it facilitates BA reabsorption. EXB is orally administered and acts locally in the gut, where it reversibly binds to IBAT to decrease the reuptake of BAs to the liver. EXB at expected therapeutic doses has minimal systemic exposure. The pharmacodynamic assessment of EXB in a Japanese phase I study showed decreased serum low-density lipoprotein cholesterol (LDL-C) levels in groups that received repeated doses of $\geq 5 \mathrm{mg} \mathrm{EXB}{ }^{8}$ In addition, a study on NAFLD mouse model reported that treatment with another IBAT inhibitor (IBATi) promoted BA biosynthesis from cholesterol, which accumulated in the liver of mice that received a high-fat diet. ${ }^{9}$ Furthermore, converting the BA pool in the liver into hydrophobic BAs decreases hepatic cholesterol accumulation via farnesoid $X$ receptor (FXR) activation, thereby improves the NAFLD status. ${ }^{9} \mathrm{EXB}$ is also expected to improve the NAFLD status as it has the same mechanism of action (MOA) and lowers the LDL-C levels. However, increased BA level in the large intestine may stimulate the secretion of water and electrolytes in the intestinal tract and movement of the gastrointestinal tract, which induces BA diarrhoea and promotes peristalsis. ${ }^{1011}$

The BA sequestrant (BAS) cholestyramine (CTM) is a strong base anion exchange resin, and its drug product alleviates hypercholesteraemia and eliminates active leflunomide metabolites from the body. CTM inhibits the absorption of exogenous cholesterol by binding to BA in the intestinal tract, which increases faeces excretion. CTM also promotes cholesterol catabolism to BA in the liver to compensate for the decreased BA level due to increased excretion. This ultimately decreases the LDL-C level. These effects are expected to promote hepatic lipid metabolism. In a Japanese clinical study, colesevelam, another BAS, improved fatty liver and liver enzymes in

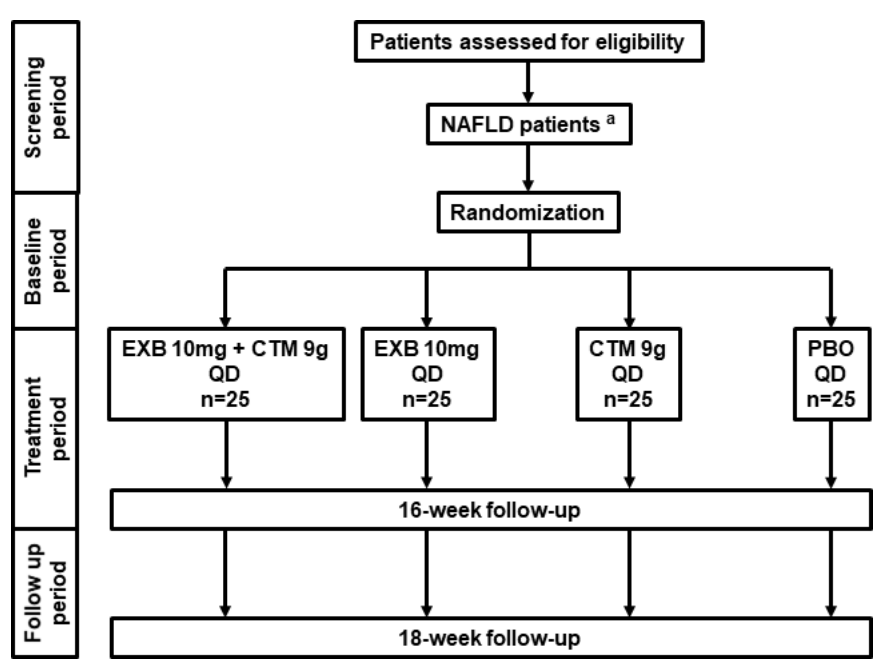

Figure 1 Study design. ${ }^{a} \mathrm{~N}=100$ enrolled. CTM, cholestyramine; EXB, elobixibat; NAFLD, non-alcoholic fatty liver disease; PBO, placebo; $\mathrm{QD}$, quaque die.

patients with NASH. ${ }^{12}$ However, the improvement in fatty liver with colesevelam treatment is not consistently recognised as an outcome because it has not been demonstrated in other studies. ${ }^{13}$ In addition to LDL-C reduction, colesevelam can alleviate BA diarrhoea, ${ }^{14}$ and a combination therapy of IBATi and BAS may prevent diarrhoea, an adverse effect of IBATi. Our study aims to investigate the efficacy and safety of EXB in combination with CTM in patients with NAFLD.

\section{MATERIALS AND METHODS \\ Trial design}

The Standard Protocol Items for Randomised Trials statement and its checklist were followed in preparing the study protocol. This trial is designed as a single-centre, randomised, double-blind, placebo-controlled, parallelgroup, investigator-initiated study to investigate the efficacy and safety of a combination therapy of $10 \mathrm{mg}$ EXB tablet and $9 \mathrm{~g}$ CTM powder formulation ( $4 \mathrm{~g}$ CTM), an EXB monotherapy $(10 \mathrm{mg})$ or a CTM monotherapy CTM (9 g), compared with those of placebo treatments. All treatments will be administered orally once daily for 16 weeks to patients with NAFLD. The experimental groups will be as follows (figure 1): the EXB +CTM group (10 $\mathrm{mg}$ EXB and $9 \mathrm{~g} \mathrm{CTM}$ ), the EXB group (10 mg EXB and CTM placebo), the CTM group (EXB placebo and $9 \mathrm{~g} \mathrm{CTM}$ ) and the PBO group (EXB placebo and CTM placebo). MRI will be performed at the baseline and 16 weeks after intervention and the data will be evaluated by a blinded independent liver specialist (KI). The study plan involves recruiting 100 adult patients with NAFLD from the Yokohama City University Hospital cohort.

\section{Study endpoints and rationale}

The primary endpoint will be to compare the absolute changes in LDL-C levels from the baseline to 16 weeks after treatment initiation among patients receiving 


\section{Table 1 Study endpoints}

\begin{tabular}{|c|c|c|}
\hline Primary endpoint & Exploratory endpoints & \\
\hline <Efficacy endpoint> & <Efficacy endpoint> & <Safety endpoint> \\
\hline \multirow{3}{*}{$\begin{array}{l}\text { Absolute change } \\
\text { from baseline in } \\
\text { serum LDL-C at } \\
\text { week } 16\end{array}$} & $\begin{array}{l}\text { Absolute change from baseline to week } 16 \text { in liver fat } \\
\text { fraction (\%) as measured by MRI-PDFF. }\end{array}$ & - Incidence of adverse events \\
\hline & $\begin{array}{l}\text { Absolute change from baseline to week } 16 \text { in hepatic } \\
\text { fibrosis as measured by MRE. }\end{array}$ & $\begin{array}{l}\text { Change from baseline in daily BSFS } \\
\text { score, no of bowel movements, and } \\
\text { PAC-QOL at every visit }\end{array}$ \\
\hline & $\begin{array}{l}\text { Change from baseline to week } 16 \text { in the following: } \\
\text { ALT, AST, GGT, HDL-C, non-HDL-C, LDL-C/HDL-C ratio }\end{array}$ & $\begin{array}{l}\text { Change from baseline in the CLDQ } \\
\text { scale at every visit }\end{array}$ \\
\hline
\end{tabular}

Class; lipid>
Change from baseline to week 16 in the following: serum
lipid fraction*, apoprotein A1 or B.

$<$ Class; Endocrine>

Change from baseline to week 16 in the following: serum c-peptide, plasma total GLP-1, plasma active GLP-1 and plasma adiponectin.

<Class; Bile acid>
Change from baseline to week 16 in the following: serum
C4, FGF-19, total bile acid, and fraction of bile acid and
faecal bile acid (total and fraction)
<Class; Inflammation>
Change from baseline to week 16 in the following: plasma
CK18 fragment M30, endotoxin activity assay, and plasma
LBP
<Class; fibrosis $>$
Change from baseline to week 16 in the following: serum
type IV collagen 7 s, PIIIP, hyaluronic acid, and TIMP-1
<Class; gut-microbiota>
Change from baseline to week 16 in faecal gut-microbiota
$<$ Class; other
$<$ Change from baseline to week 16 in serum/faecal

choline, trimethylamine, TMAO, amino acids.
Change from baseline to week 16 in faecal short chain
fatty acids.

All objectives will be compared among the EXB $(10 \mathrm{mg})$ and CTM $(9 \mathrm{~g})$ combination therapy, EXB $(10 \mathrm{mg})$ or CTM $(9 \mathrm{~g})$ monotherapy, and placebo groups.

${ }^{*}$ Chylomicron-cholesterol, chylomicron-TG, intermediate density lipoprotein cholesterol, very low-density lipoprotein cholesterol (VLDL-C), LDL-C, HDL-C, LDL-TG, VLDL-TG.

ALT, alanine transaminase; AST, aspartate transaminase; BSFS, Bristol Stool Form Scale; CLDQ, Chronic Liver Disease Questionnaire; CTM, cholestyramine; EXB, elobixibat; FGF-19, fibroblast growth factor 19; GLP-1, glucagon-like peptide-1; HDL-C, high-density lipoprotein cholesterol; LBP, lipopolysaccharide-binding protein; LDL-C, low-density lipoprotein cholesterol; MRE, MR elastography; PAC-QOL, Patient Assessment of Constipation Quality of Life; PDFF, proton density fat fraction; PIIIP, procollagen-3-peptide; TG, triglyceride; TIMP-1, tissue inhibitor of metalloproteinase-1; TMAO, trimethylamine $\mathrm{N}$-oxide.

EXB-CTM combination therapy, EXB monotherapy, CTM monotherapy and placebo (table 1 ). Recent studies reported that the serum and hepatic BA levels increase in patients with NASH and that the severity of NASH is related to a specific change in enterohepatic BA circulation. ${ }^{6}$ As the inhibition of BA reabsorption from the intestine is suggested to possibly improve NASH, we will focus on LDL-C reduction associated with increased BA production. LDL-C is commonly used for assessing NAFLD. For instance, phase II studies of VK2809 and obeticholic acid used LDL-C as the primary endpoint, ${ }^{15}$ and a phase IIa study on EXB monotherapy for NAFLD in the USA used LDL-C as the primary endpoint. The NAFLD guidelines recommend using antidyslipidaemic drugs to treat NAFLD. Because reducing the LDL-C level may improve cholesterol accumulation in the liver and is expected to improve NAFLD, LDL-C was considered an appropriate endpoint for monitoring NAFLD. As CTM, which is 
proposed to be used in the present study, is a hypercholesterolaemia treatment, and EXB is shown to reduce LDL-C in patients with chronic constipation, LDL-C is expected to be a sensitive surrogate for monitoring the efficacy of the EXB-CTM combination therapy.

As we aim to confirm the proof of concept (POC) in this phase IIa study, it is appropriate to monitor treatment efficacy using LDL-C as a surrogate marker that can be tested within a short period rather than histopathological assessment using a more invasive liver biopsy. The exploratory endpoints include liver function and liver fat fraction assessments that will provide a comprehensive evaluation. After confirming the POC in this study, we plan to perform drug-dosing studies in the next phase, including liver biopsy analysis for determining the NASH status and hepatic fibrosis after long-term drug administration.

Our current exploratory endpoint will determine the absolute change in the liver fat fraction and hepatic fibrosis, measured by MRI-proton density fat fraction (PDFF) and MR elastography (MRE) according to our previous method, ${ }^{16}{ }^{17}$ respectively, from the baseline to 16 weeks after treatment initiation. The other parameters that will be monitored as exploratory endpoints for the absolute changes between the baseline and 16 weeks after treatment initiation include alanine aminotransferase (ALT), aspartate aminotransferase, $\gamma$-glutamyl transpeptidase, high-density lipoprotein cholesterol (HDL-C), non-HDL-C, LDL-C/HDL-C ratio and triglyceride. Other variables monitored will be adverse events (AEs), standard laboratory analysis results, physical examination, vital signs and compliance rate. Physical assessments will be performed and evaluated at Yokohama City University, using standard procedures.

\section{Rationale for treatment dose, mode and duration}

We considered it was appropriate to use the currently approved dose of each drug in our study to investigate the effects of the EXB-CTM combination therapy in an exploratory manner and provide an adequate and safe experience under clinical management. Although not previously published or presented at academic meetings, this combination therapy at the approved dosing is widely known to improve hepatic parameters and follow-up without adverse drug reactions, including diarrhoea and constipation, in patients with NAFLD in clinical settings in Japan. The approved dosing should be sufficient to ensure safety and tolerability in patients with NAFLD and allow an efficacy evaluation of the combination therapy.

Although the hypercholesterolaemia regimen is administered at $9 \mathrm{~g} /$ dose, 2-3 times daily, the dose in this study will be administered once daily before breakfast concomitant with EXB. This regimen is based on the consideration that a combination therapy is preferable for achieving continuous drug compliance, along with reducing AEs due to EXB, including abdominal pain and diarrhoea.

Global exploratory NAFLD trials have adopted a treatment period of at least 12 weeks (Safety, Tolerability,
Pharmacokinetics and Efficacy of LMB763 in Patients With NASH: NCT02913105; A Study of the Efficacy and Safety of CF102 in the Treatment of Non-Alcoholic Fatty Liver Disease: NCT02927314). A Japanese phase III study of EXB in patients with chronic constipation demonstrated that the LDL-C level rapidly decreased after two treatment weeks, and lower levels were maintained for at least 52 weeks. ${ }^{7}$ However, in this study, we plan to use MRI examination as an exploratory endpoint, and it requires a minimum treatment period for efficacy determination. Thus, a 16-week treatment period was incorporated into the study design to ensure an appropriate EXB efficacy evaluation.

\section{Drug supply}

Only the Patient Enrolment Centre will know the treatment allocation, and double blinding of the physicians and patients will be maintained throughout the study until all patients have completed the 16 weeks study, and the database has been locked for all study data. Tablets of EXB (5 mg) and the corresponding reference placebo, which is indistinguishable in appearance, were manufactured and supplied by EA Pharma (Tokyo, Japan). The CTM powder, $9 \mathrm{~g}$ /dose (CTM $4 \mathrm{~g}$ ), was purchased from the market, and its reference placebo, which is indistinguishable in appearance, was manufactured; both were supplied by TOYO Pharmaceutical (Osaka, Japan). For the study drugs prescribed, the physicians will enter the drug allocation number provided by the patient enrolment centre on the prescription form. The drug manager will dispense the study drug to the patient with the drug allocation number. Exceptions to blinding will be to secure a patient's safety and for treatment on request to the patient enrolment centre personnel by the principal investigator.

\section{Sample size estimation}

Due to the exploratory nature of this study, formal power calculations were not used to determine sample sizes. The number of subjects (100 patients in total, 25 patients per treatment group) was chosen based on the data from a Japanese phase III clinical study and a long-term study on EXB in patients with chronic constipation. ${ }^{7}$ The phase III study showed an LDL-C level reduction after 2 weeks of treatment, whereas the long-term study demonstrated that similar reductions persisted from week 4 to week 52 of treatment. Hence, we hypothesise that the reduction in the LDL-C level associated with EXB treatment (10 $\mathrm{mg}$ ) will remain the same between weeks 2 and 16 . As the mean difference in the LDL-C level from the baseline between the $10 \mathrm{mg}$ EXB group and placebo group was $-15.7 \mathrm{mg} / \mathrm{dL}$, the pooled SD was predicted as 17.9 $\mathrm{mg} / \mathrm{dL}$. Using sample sizes of $\mathrm{n}=20$ per group, the study has $80 \%$ power with two-sided $\alpha=0.05$. Taking protocol violations and dropouts into account, a sample size of 25 patients in each group was calculated to compare EXB and placebo groups. Assuming that there is no synergistic effect of EXB and CTM, the powers to compare (1) CTM 
use and CTM no-use groups, and (2) EXB+CTM and placebo groups will be large enough.

\section{ELIGIBILITY}

The physicians will enter legally capable patients into the Screening List, assign an identification code to each patient, and determine eligibility according to the inclusion and exclusion criteria (table 2 ). We will include only patients aged $\geq 20$ and $<75$ years after obtaining informed consent. This is because (1) the legal adult age to obtain consent is 20 years in Japan and (2) patients over 75 years of age generally have impaired physiological function and are more prone to AEs. If no eligibility issues are identified, the investigator or subinvestigator and investigative staff will enter the necessary information into the electronic data capture (EDC) system for enrolment. The patient enrolment number will then be assigned, and enrolment will be completed.

\section{Randomisation and masking}

The patients will be randomised to each group (EXB+CTM, EXB, CTM and PBO) at a ratio of 1:1:1:1 using a computer-generated centrally administered procedure (no factor for stratification). The contract research organisation will create the list of study drug randomisation and link the appropriate study drug number. After the investigators confirm the eligibility of participants, the required information will be entered in the EDC system, and the drug number will be issued. Investigators and patients will be blinded to the details of the assignment to conceal the drug allocation number in independent contact research organisation until the key open. All trial drugs will be packed identically and identified only by the number assigned. As noted above, the treatment assignments will be fully masked from the patients and physicians.

\section{KEYCODE BREAK}

If the investigator or subinvestigator considers it urgently necessary to break the study keycode prematurely, they will contact the person responsible for study drug randomisation to file the request. This may occur due to a serious $\mathrm{AE}$ (SAE), the need for treating an $\mathrm{AE}$ or a similar situation.

\section{Harms and AE monitoring}

AEs are defined as any unfavourable or unintended sign (including laboratory parameters and abnormal vital signs), symptom, or disease that may occur during the study period. AEs that are not directly related to the study drugs may develop. The investigator or subinvestigator will assess the severity of the AEs. Any AE that meets any of the following criteria will be considered an SAE: death, lifethreatening, hospitalisation requirement or prolonged hospitalisation for treatment, disability, disability threat, other serious conditions, congenital disease or anomaly in offspring. If an SAE occurs, the investigator or subinvestigator will appropriately treat it, and the investigator will immediately report the details to the hospital director and the study drug supplier.

\section{STUDY PROCEDURES}

The investigator or subinvestigator will perform all observations, tests, investigations and evaluations according to the descriptions provided in table 3 . If a blood test is scheduled at a study visit, blood will be collected only after the patient had fasted for at least 8 hour. After the start of treatment, drug returns and blood test results will be checked to monitor adherence in each visit. Blood/ stool sampling will be collected and stored for the exploratory analysis of genes (single nucleotide polymorphisms; patatin-like phospholipase domain containing three and transmembrane 6 superfamily member 2 ), fibrosis and inflammation, on obtaining consent separately. When the study drug is given to the participants at each visit, the pharmacist will provide instructions on the dosage and administration. At the next visit, the pharmacist will ask the participants to bring the unused study drug in their next visit and record the number of tablets (packages) that are returned. These strategies will improve adherence to the intervention protocol.

\section{CONCOMITANT TREATMENT}

The administration of the following medications and therapy are prohibited from the time of Screening to the end of follow-up: BA agents (ursodeoxycholic acid, chenodeoxycholic acid and dehydrocholic acid), IBATis, anion-exchange resin agents other than the study drugs, thiazolidinedione and bariatric surgery. The restricted concomitant medications are permitted only if they are continuously used at a stable dose within 4 weeks of the study enrollment. The dose and dosage regimen of the following drugs must be determined to be within the stable range at the start of study treatment and kept stable until the end of study treatment: antihypertensive drugs (angiotensin II receptor blockers only); vitamin E; antidyslipidemic drugs and antidiabetic drugs (dipeptidyl peptidase-4 inhibitors, GLP-1 receptor agonists and insulin injections).

\section{Criteria and procedure for withdrawal from the study}

The investigator or subinvestigator will discontinue the study enrollment of a patient if the participant meets any of the following criteria: (1) the patient desires withdrawal, (2) after enrolment, the patient is found not to meet the inclusion criteria or exclusion criteria, (3) if the investigator or subinvestigator opines that having the patient continue in the study is not appropriate due to an $\mathrm{AE}$, and (4) if the investigator or subinvestigator opines that having the patient continue in the study is not appropriate due to any other reason. 
Table 2 Patient inclusion and exclusion criteria

\section{Inclusion criteria}

1 Patients who received adequate explanation about this study and provided written informed consent

2 Patients who are $\geq 20$ and $<75$ years of age at the time of informed consent

3 Patients who have a current biopsy-confirmed NASH within 8 months of screening or a suspected diagnosis of NAFLD/NASH based on the criteria outlined below:

(1) Biopsy-confirmed NASH is defined as histological NASH diagnosis with fibrosis stage F1 through F3 and a NAFLD activity score (NAS) of $\geq 4$ with a score of $\geq 1$ in each of the NAS components below as assessed by a pathologist using the NASH Clinical Research Network criteria:

1. Steatosis (scored $0-3$ )

2. Ballooning degeneration (scored $0-2$ )

3. Lobular inflammation (scored 0-3)

(2) The suspected diagnosis of NAFLD/NASH is based on the

following criteria:

1. AST $\geq 20 \mathrm{U} / \mathrm{L}$ and $A L T \geq 40 \mathrm{U} / \mathrm{L}$ in males or $\geq 28 \mathrm{U} / \mathrm{L}$ in females

2. Waist circumference $\geq 85 \mathrm{~cm}$ in males or $\geq 90 \mathrm{~cm}$ in females

a. Diagnosis of metabolic syndrome having two or more of the following three risk factors at screening:Fasting plasma glucose $\geq 110 \mathrm{mg} / \mathrm{dL}$ or undergoing drug treatment for elevated glucose

b. SBP $\geq 130 \mathrm{~mm} \mathrm{Hg}$ and/or $\mathrm{DBP} \geq 85 \mathrm{~mm} \mathrm{Hg}$ or undergoing drug treatment for hypertension, or antihypertensive drug treatment in a patient with a history of hypertension

c. TGs $\geq 150 \mathrm{mg} / \mathrm{dL}$ or undergoing drug treatment for elevated TGs, and/or HDL-C $<40 \mathrm{mg} / \mathrm{dL}$ or undergoing drug treatment for reduced HDL-C

4 Screening MRI -PDFF with $\geq 8 \%$ liver steatosis

\section{Exclusion criteria}

Women who are pregnant, breast feeding, possibly pregnant or do not agree to use birth control during the study.

$\mathrm{BMl}<23 \mathrm{~kg} / \mathrm{m}^{2}$.

Liver stiffness measured by MRE $>6.7 \mathrm{kPa}$.

Any of the following laboratory abnormalities:

1. $A L T>5 \times U L N$ or $A S T>5 \times U L N$.

2. PT-INR $\geq 1.3$ unless on anticoagulant therapy.

3. Total bilirubin $>U L N$, except with an established diagnosis of Gilbert's syndrome.

4. Platelet count $<80000 / \mu \mathrm{L}$.

5. eGFR $<45$ as calculated by the BSA adjustment (normalised eGFR).

5 Fasting serum LDL-C $>120 \mathrm{mg} / \mathrm{dL}$ or receiving antidyslipidemic drugs

Acute or chronic liver disease other than NAFLD/NASH including but not limited to the following:

(1) Hepatitis B (as defined by the presence of HB antigen at screening) or hepatitis $\mathrm{C}$ (as defined by the presence of HCV antibody(anti-HCV))

Patients with positive anti-HCV who test negative for HCV ribonucleic acid (HCV-RNA) at screening will be allowed to participate in the study as long as there is evidence of viral negativity for a minimum of 12 months before screening.

1. Evidence of $\mathrm{AlH}$.

2. History of PBC, PSC, Wilson's disease, alpha-1-anti-trypsin deficiency, hemochromatosis or iron overload, drug-induced ALD, or known bile duct obstruction.

3. Suspected or proven HCC.

6 Be willing to maintain a stable diet and physical activity Known history of HIV.

throughout the course of the study

$\begin{array}{ll}7 & \text { Medical history of liver cirrhosis. }\end{array}$

Continued 
Table 2 Continued

\section{Inclusion criteria}

10

11

12

13

14

15

16

\section{Exclusion criteria}

Use of the following medications:

1. GLP-1 agonists unless on a stable dose 3 months before screening or liver biopsy.

2. Ursodeoxycholic acid or thiazolidinediones within 3 months before screening.

3. Antidyslipidemic drugs have been stable for $\geq 3$ months before screening.

4. Oral antidiabetic drugs have been stable for $\geq 3$ months before screening.

5. Agents (including herbal over-the-counter weight loss preparations) or medications known to significantly affect body weight within 3 months before screening.

History of significant alcohol consumption, defined as an average of $\geq 20 \mathrm{~g} /$ day in female patients and $\geq 30 \mathrm{~g} /$ day in male patients, for a period of $>3$ consecutive months within 1 year before screening, hazardous alcohol use (Alcohol Use Disorders Identification Test score $\geq 8$ ), or an inability to reliably quantify alcohol consumption but determined as alcohol polydipsia based on the judgement of the investigator or subinvestigator.

Weight change of $\geq 10 \%$ within the 6 months before screening or $\geq 5 \%$ within the 3 months before screening.

Surgery planned during the study period or after bariatric surgery (eg, gastroplasty and Roux-en-Y gastric bypass).

Type 1 diabetes by medical history.

Uncontrolled type 2 diabetes defined as haemoglobin A1c (HbA1c) of $>9.5 \%$ at screening (patients with $\mathrm{HbA} 1 \mathrm{c}>9.5 \%$ may be rescreened) or requiring insulin dose adjustment of $>10 \%$ within 2 months before screening.

Clinical hyperthyroidism or hypothyroidism or screening hormone results pointing to thyroid dysfunction.

Patients receiving dose-stable thyroid replacement therapy for $\geq 3$ months before screening will be allowed to participate in this study as long as thyroid tests show that the patient is euthyroid and stable.

History of any condition causing malabsorption such as chronic pancreatitis, extensive bowel/small intestine surgery, coeliac disease or bile flow obstruction.

18

History of any condition associated with acute or chronic diarrhoea such as IBD, functional diarrhoea, IBS with predominant diarrhoea, IBS with mixed bowel habits or unclassified IBS.

19

20

21

22

23

24

25

26
Uncontrolled hypertension (either treated or untreated) defined as SBP of $>160 \mathrm{~mm} \mathrm{Hg}$ or a DBP of $>100 \mathrm{~mm} \mathrm{Hg}$ at screening.

A history of New York Heart Association Class III or IV heart failure, or known left ventricular ejection fraction of $<30 \%$.

A history of myocardial infarction, unstable angina, percutaneous coronary intervention, coronary artery bypass graft, or stroke or major surgery within 6 months prior before screening.

Active substance abuse, within 1 year before screening.

Participation in an investigational new drug trial in the 30 days before screening or within five half-lives of an investigational agent, whichever is longer.

Complication with malignancy

Patients with a history of malignancies that have been treated with a curative intent or completed chemotherapy may be eligible patients under evaluation for malignancy are not eligible.

Known intolerance to MRI or conditions contraindicated for MRI procedures.

Any other condition which is considered to be inappropriate for the study by the Investigator or subinvestigator.

Continued 
AlH, autoimmune hepatitis; ALD, alcoholic liver disease; ALT, alanine aminotransferase; AST, aspartate aminotransferase; BMI, body mass index; BSA, body surface area; DBP, diastolic blood pressure; eGFR, estimated glomerular filtration rate; GLP-1, glucagon-like peptide-1; HBS, hepatitis B surface; HCC, hepatocellular carcinoma; HCV, hepatitis C virus; HDL-C, high-density lipoprotein cholesterol; IBD, inflammatory bowel disease; IBS, irritable bowel syndrome; LDL-C, low-density lipoprotein cholesterol; MRE, MR elastography; NAFLD, non-alcoholic fatty liver disease; NAS, NAFLD activity score; NASH, non-alcoholic steatohepatitis; PBC, primary biliary cholangitis; PDFF, proton density fat fraction; PSC, primary sclerosing cholangitis; PT-INR, prothrombin time-international normalised ratio; SBP, systolic blood pressure.

\section{EFFICACY EVALUATION}

The primary efficacy endpoint will be the absolute change in LDL-C level from the baseline to 16 weeks after treatment initiation. The exploratory endpoints are provided in table 1 . The assessment of the MRI-PDFF/MRE will be performed by an independent liver specialist who is blinded to the treatment (KI).

\section{SAFETY ASSESSMENTS}

The following safety evaluations will be performed during each patient visit from the time of treatment initiation until the 2 weeks follow-up period: (1) incidence of AEs in the treatment groups compared with that of the placebo groups, (2) change in the daily score of the Bristol Stool Form Scale from the baseline regarding the number of bowel movements and the Patient Assessment of Constipation Quality of Life, (3) change in the Chronic Liver Disease Questionnaire scale from the baseline.

\section{POPULATION ANALYSIS}

The set of subjects to be analysed will be determined before locking the data of each patient and will be defined as follows. The modified intention to treat, which is the full analysis set (FAS), and per-protocol set (PPS) will be used for the assessment of primary efficacy. The FAS will include all patients who are randomised, except those who meet any of the following criteria: (1) patients with major protocol deviation (eg, deviation of informed consent and major deviation in the study procedures), (2) patients who have not received any dose of the study drugs and (3) patients who have no measurement of the efficacy endpoint. A PPS will include patients without protocol deviations. The safety analysis set will be used for safety assessment and will include all patients who receive at least one dose of the study drug.

\section{STATISTICAL ANALYSIS}

Considering the placebo-controlled randomised parallelgroup comparison design, we will use descriptive statistics to summarise differences between the placebo group and each monotherapy or combination group and those between each monotherapy group and the combination group. Unless otherwise noted, the descriptive statistics include the number of patients, mean, SD, median, minimum and maximum values of continuous variables, frequency and percentage of categorical variables.
For the primary endpoint, the change in serum LDL-C from the baseline to week 16 (difference in measurement values between time points) will be summarised for each treatment group. Point estimates and confidence intervals for the mean difference from the baseline between the placebo group and each monotherapy or combination group and those between each monotherapy group and the combination group will be calculated. The changes over time in the measured parameters will be summarised for each treatment group. The ratio of change (ratio of the absolute change to the baseline value) will also be analysed using the same methods. The exploratory endpoints will be analysed similar to the primary endpoint. Due to the exploratory nature of this study, a concrete statistical hypothesis is not formulated. For exploratory purposes, in case any statistical test is performed or CI of any parameter is calculated, the significance level of 5\% (two sided) and the confidence coefficient of $95 \%$ will be used. Multiplicity arising from the interpretation of statistical tests or confidence intervals will not be adjusted.

\section{INTERIM ANALYSIS}

Not applicable.

\section{Data management, central monitoring and audit}

The investigators' sites will maintain individual records of each patient as source data, which include a copy of informed consent, medical records, laboratory data and other records or notes. All data will be collected by the independent data management centre. The data management centre will oversee the interstudy data sharing process. The clinical data entry, data management and central monitoring will be performed using the electric data capture VIEDOC 4 (PCG Solutions, Sweden). Furthermore, auditing will be planned at an external clinical research organisation.

\section{Efficacy and safety assessment board}

Although no interim analysis will be performed in this study, this committee was established to monitor the overall study focusing on safety, as this is the first study to administer EXB and CTM in combination to patients with NAFLD. The board will consist of three external board members who have no relationship with the operations involved in the study. 
Table 3 Schedule of observations, tests and assessments

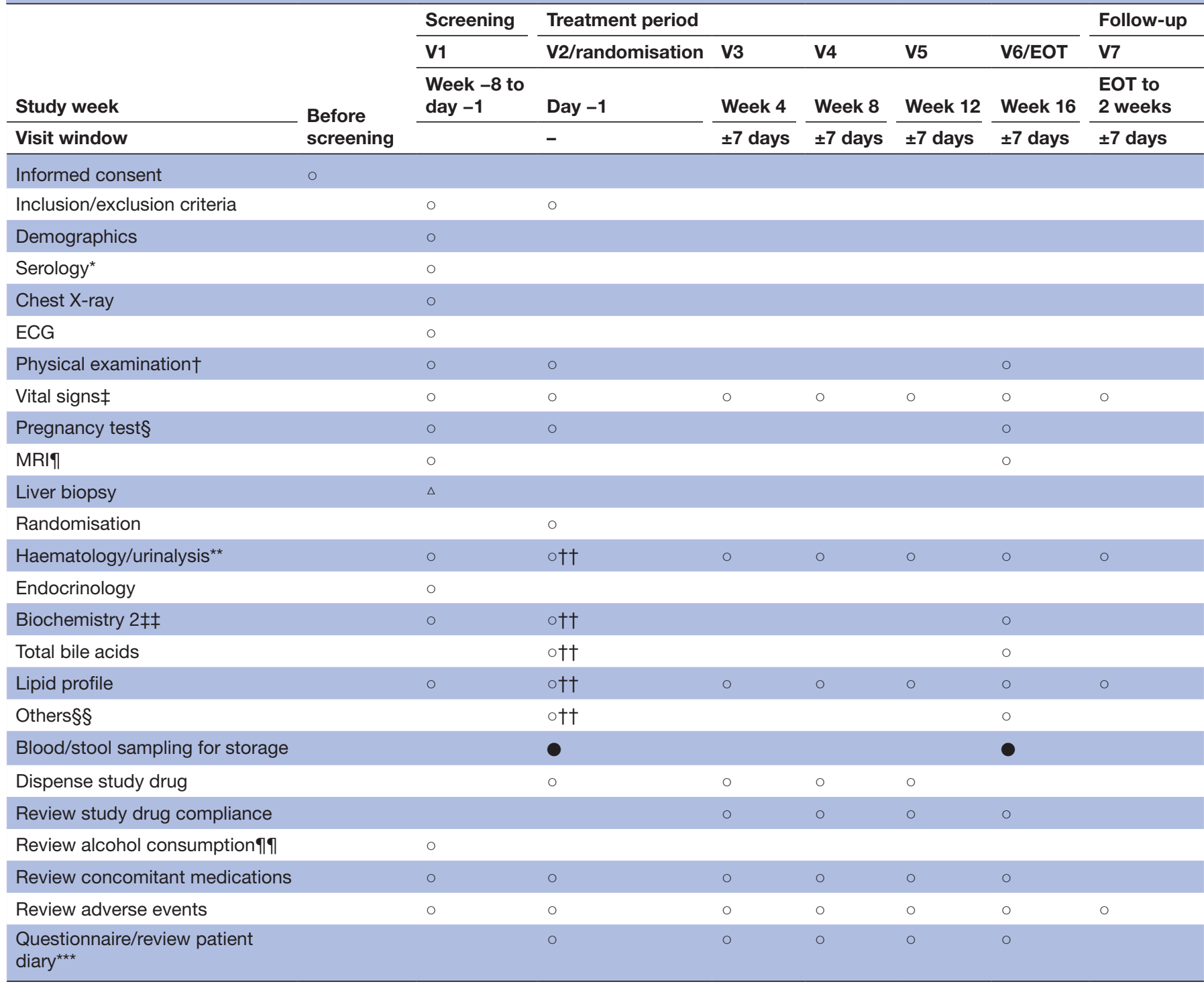

$\circ$, To be performed.

$\triangle$, Information will be collected from patients who have liver biopsy results.

- Blood/stool sampling for storage will be collected for the analysis of genes, fibrosis, and inflammation etc, upon obtaining consent separately. *Includes HB antigen, HCV antibody and HCV-RNA.

tIncludes height (at V1 only), body weight, waist circumference and waist-to-hip ratio. BMI will be calculated based on height and weight.

$\ddagger V$ ital signs include blood pressure, heart rate, respiratory rate and axillary temperature.

§For women of childbearing potential, a urinary pregnancy test will be performed at V1, V2 and V6. The test is not required at V2 when it occurs within 1 month after V1.

IPatients will undergo an MRI to measure liver fat (PDFF) and total liver volume. Patients who discontinue before V6 (week 16) should undergo an $\mathrm{MRI}$ at the end of treatment if they completed at least 4 weeks of treatment.

${ }^{* *}$ Haematology/urinalysis includes haematology/coagulation, biochemistry 1 and urinalysis.

†† Data within 1 month can be used as a substitute.

‡¥Biochemistry 2 includes glucose, HbA1c and insulin.

$\S \S 0$ thers include high-sensitivity CRP, type IV collagen 7 s, FIB-4 and APRI.

१ी

${ }^{* * *}$ Questionnaires of PA-QOL and CLDQ will be answered at each visit. Number of bowel movements and BS score will be collected from the patient diary.

APRI, aspartate aminotransferase -to-platelet ratio index; BMI, body mass index; BS, bristol stool; CLDQ, Chronic Liver Disease Questionnaire; CRP, C reactive protein; FIB4, fibrosis-4; HbA1c, haemoglobin A1c; HCV, hepatitis C virus; PA-QOL, Patient Assessment of Constipation Quality of Life;

PDFF, proton density fat fraction.

\section{Study flow and schedule of enrolment, interventions and assessments}

A study flow chart is shown in figure 1 . The study schedule is presented in table 3 .

\section{Patient and public involvement}

In this randomised controlled trial, patients will be involved in the recruitment to and conduct of the study. Especially, the development of the research question and 
outcome measures were based on patients' priorities, experience and preferences. The results of this study will be disseminated by email to the participants who indicate that they want the results. The burden of intervention will be assessed by patients before the commencement of the trial; patients' satisfaction of the treatment will be collected as a part of the post-intervention assessment.

\section{Ethics and dissemination}

This study will be conducted in compliance with the Declaration of Helsinki, 'Order for Enforcement of the Act on Securing Quality, Efficacy and Safety of Products Including Pharmaceuticals and Medical Devices,' and Good Clinical Practice standards. The study protocol and relevant supporting data were approved on 26 November 2019 by the institutional ethics committee before participant enrolment. The trial results will be reported according to the Consolidated Standards of Reporting Trials 2010 guidelines. Written informed consent (see online supplementary file 1 for informed consent form) for study participation will be obtained from all enrolled participants. The amended or modified study protocol will be approved by the IRB. The results of this study will be submitted for publication in international peerreviewed journals, and the key findings will be presented at conferences. The funder has no role in the study design, data collection or data analysis. Participants will be informed of the results of the trial by the investigators. Authorship will be ascribed in accordance with the International Committee of Medical Journal Editors guidance.

\section{DISCUSSION}

This is the first POC study proposed to explore the effect of EXB in combination with CTM in patients with NAFLD. A previous study showed that an IBATi improved both hepatic and whole-body aspects in a mouse model of NAFLD ${ }^{9}$ However, EXB causes AEs, including abdominal pain and diarrhoea, by increasing colonic BAs, resulting in the development of BA-induced diarrhoea. Therefore, the safety and tolerability of monotherapy with EXB might decrease in patients with NAFLD. Considering the MOA, the removal of excess BAs from the colon using CTM has the potential to improve BA-induced diarrhoea.

In some well-known trials (eg, PIVENS, FLINT and GOLDEN),${ }^{15}{ }^{18-20}$ the primary endpoints included liver histology, which was evaluated using liver biopsy specimens. Liver histology endpoints, such as the complete resolution of NASH, are considered surrogates for preventing cirrhosis because they are considered predictive of clinical benefit but are not direct measures. Currently, the only method to diagnose NASH is a liver biopsy, as described in the guidelines ('gold standard'). However, in Japan, the number of patients with NAFLD exceeds 10 million, and it is difficult to perform liver biopsy for all NAFLD patients. In addition, liver biopsy has various problems such as invasiveness, cost, and differences in interpretation among pathologists.
In recent years, the progress of MRI technology has been remarkable, and it has become possible to accurately diagnose steatosis and fibrosis using MRE. ${ }^{21}{ }^{22}$ Quantification of body fat percentage using MRI is also possible by using echo asymmetry and least-squares estimation sequence methods to measure PDFF by repeated decomposition of water and fat. ${ }^{1723}$ MRE and MRI-PDFF can be performed at the same time in one imaging, and the combined results can evaluate liver steatosis and liver fibrosis. Non-invasive evaluation of liver fibrosis and liver steatosis using MRI has the potential to replace liver biopsy in the clinical setting. ${ }^{17}$ Hence, we chose MRI for the noninvasive assessment of hepatic steatosis in this study. Quantification of liver fat content using MRI-PDFF has been previously shown to be sensitive in detecting changes, and it has been used in NASH clinical trials for quantitative fat assessment. ${ }^{1721}$

Our study has the following strengths: (1) the first randomised, placebo-controlled, double-blinded study focused on removing BAs in patients with NAFLD; (2) a novel POC study considering the drug MOA for improving the treatment effect and tolerability, as well as reducing the AEs; (3) MR images will be captured following a standardised protocol and processed under the supervision of a hepatoradiologist blinded to the study; (4) the comparison of colocalised regions of interest for fat content changes in each of the nine liver segments between weeks 0 and 16 ; (5) the final assessment of the MRI examinations will be randomised with regard to time points in order to reduce bias and (6) the measurement of exploratory endpoints such as the lipid class, endocrine functions, BA, inflammation, fibrosis, gut microbiota and trimethylamine-N-oxide (table 2). Nevertheless, our study also has the following limitations: (1) will be performed in a single centre; (2) a relatively small sample size; (3) a relatively short treatment duration; and (4) the lack of liver biopsy.

NAFLD/NASH is a heterogeneous disease with correspondingly complex pathophysiology, which includes redundant pathways that may not be uniform among patients. Considering the MOA of pharmaceuticals for treating NAFLD/NASH, its complex pathophysiology permits the development of a wide array of potentially viable therapeutic targets, especially components of antimetabolic, anti-inflammatory and antifibrotic pathways. ${ }^{24}$ In a phase IIa trial for NASH/NAFLD therapies, BMS-986036 (FGF21) ${ }^{25}$ and NGM282 (FGF19) ${ }^{26}$ exerted an antimetabolic effect, and the liver fat content was used as the primary endpoint. Although the current NAFLD therapy focuses on antimetabolic, anti-inflammatory and antifibrotic aspects in the liver as MOA, decreasing BAs for treating NAFLD is a novel therapeutic target for patients with NAFLD. EXB-CTM combination therapy-associated improvements in the changes of the absolute and relative liver fat levels changes are clinically relevant. 


\section{Author affiliations}

${ }^{1}$ Department of Gastroenterology and Hepatology, Yokohama City University Graduate School of Medicine, Yokohama, Japan

${ }^{2}$ Department of Palliative Medicine, Yokohama City University Hospital, Yokohama, Japan

${ }^{3}$ Department of Biostatistics, Yokohama City University Graduate School of Medicine, Yokohama, Japan

${ }^{4}$ Department of Pharmacology, Shimane University Faculty of Medicine Graduate School of Medicine, Izumo, Shimane, Japan

Acknowledgements We like to thank Editage (https://editage.jp) for editing a draft of this manuscript and helping to draft the abstract. We thank in advance all patients, patient advisers, staffs, and investigators who will be involved in this study.

Contributors TKe and AN participated in study design. TKe and TKo conducted feasibility phase work. Recruitment of participants and follow-up will be performed by TKe, AO, Ml, TKo, YH, YO, MY and SS. Reading of MRI will be done by Kl. Exploratory item will be measured by HU and KW. TKe, TKo, YS, KY, TY and AN will participate in data interpretation. All authors contributed to writing, and all read and approved the final manuscript.

Funding This study is sponsored by Yokohama City University and funded by EA Pharma (Tokyo, Japan).

Competing interests Data will be retained in accordance with the Japanese ethical guidelines for clinical research. Participants will be allocated a unique identification (ID) number at entry. The master list linking participant personal information and ID number will be maintained in a separate locked cabinet and password-protected hard drive. Data will be analysed by ID number only. Records will be retained for 5 years after study completion, and then destroyed by the data center. AN reports grants and research support from Gilead, Mylan EPD, EA Pharma, Kowa, Taisho, Biofermin; is a consulting adviser for Gilead, Boehringer Ingelheim, BMS, Kowa, Astellas, EA Pharma, Mylan EPD. Other authors declare no competing interests.

Patient and public involvement Patients and/or the public were involved in the design, or conduct, or reporting, or dissemination plans of this research. Refer to the Methods section for further details.

\section{Patient consent for publication Obtained.}

Ethics approval The Ethics Committee of Yokohama City University Hospital approved the study protocol on 26 November 2019.

Provenance and peer review Not commissioned; externally peer reviewed.

Data availability statement Data are available upon reasonable request. All data relevant to the study are included in the article or uploaded as online supplementary information.

Open access This is an open access article distributed in accordance with the Creative Commons Attribution Non Commercial (CC BY-NC 4.0) license, which permits others to distribute, remix, adapt, build upon this work non-commercially, and license their derivative works on different terms, provided the original work is properly cited, appropriate credit is given, any changes made indicated, and the use is non-commercial. See: http://creativecommons.org/licenses/by-nc/4.0/.

\section{ORCID iDs}

Takaomi Kessoku http://orcid.org/0000-0002-5587-1386

Atsushi Nakajima http://orcid.org/0000-0002-6263-1436

\section{REFERENCES}

1 Eguchi $\mathrm{Y}$, Hyogo $\mathrm{H}$, Ono $\mathrm{M}$, et al. Prevalence and associated metabolic factors of nonalcoholic fatty liver disease in the general population from 2009 to 2010 in Japan: a multicenter large retrospective study. J Gastroenterol 2012;47:586-95.

2 Day CP. Natural history of NAFLD: remarkably benign in the absence of cirrhosis. Gastroenterology 2005;129:375-8.

3 Musso G, Gambino R, Cassader M. Cholesterol metabolism and the pathogenesis of non-alcoholic steatohepatitis. Prog Lipid Res 2013;52:175-91.

4 Ferslew BC, Xie G, Johnston CK, et al. Altered bile acid metabolome in patients with nonalcoholic steatohepatitis. Dig Dis Sci 2015;60:3318-28.
5 Aranha MM, Cortez-Pinto H, Costa A, et al. Bile acid levels are increased in the liver of patients with steatohepatitis. Eur $J$ Gastroenterol Hepatol 2008;20:519-25.

6 Puri P, Daita K, Joyce A, et al. The presence and severity of nonalcoholic steatohepatitis is associated with specific changes in circulating bile acids. Hepatology 2018;67:534-48.

7 Nakajima A, Seki M, Taniguchi S, et al. Safety and efficacy of elobixibat for chronic constipation: results from a randomised, double-blind, placebo-controlled, phase 3 trial and an openlabel, single-arm, phase 3 trial. Lancet Gastroenterol Hepatol 2018;3:537-47.

8 Kumagai Y, Amano H, Sasaki Y, et al. Effect of single and multiple doses of elobixibat, an ileal bile acid transporter inhibitor, on chronic constipation: a randomized controlled trial. Br J Clin Pharmacol 2018;84:2393-404.

9 Rao A, Kosters A, Mells JE, et al. Inhibition of ileal bile acid uptake protects against nonalcoholic fatty liver disease in high-fat diet-fed mice. Sci Trans/ Med 2016;8:357ra122.

10 Hofmann AF. The syndrome of ileal disease and the broken enterohepatic circulation: cholerheic enteropathy. Gastroenterology 1967;52:752-7.

11 Sadik R, Abrahamsson H, Ung K-A, et al. Accelerated regional bowel transit and overweight shown in idiopathic bile acid malabsorption. Am J Gastroenterol 2004;99:711-8.

12 Taniai M, Hashimoto E, Tobari M, et al. Treatment of nonalcoholic steatohepatitis with colestimide. Hepatol Res 2009;39:685-93.

13 Le T-A, Chen J, Changchien C, et al. Effect of colesevelam on liver fat quantified by magnetic resonance in nonalcoholic steatohepatitis: a randomized controlled trial. Hepatology 2012;56:922-32.

14 Mottacki N, Simrén M, Bajor A. Review article: bile acid diarrhoea pathogenesis, diagnosis and management. Aliment Pharmacol Ther 2016;43:884-98.

15 Neuschwander-Tetri BA, Loomba R, Sanyal AJ, et al. Farnesoid X nuclear receptor ligand obeticholic acid for non-cirrhotic, nonalcoholic steatohepatitis (Flint): a multicentre, randomised, placebocontrolled trial. Lancet 2015;385:956-65.

16 Patel J, Bettencourt R, Cui J, et al. Association of noninvasive quantitative decline in liver fat content on MRI with histologic response in nonalcoholic steatohepatitis. Therap Adv Gastroenterol 2016;9:692-701.

17 Imajo K, Kessoku T, Honda Y, et al. Magnetic resonance imaging more accurately classifies steatosis and fibrosis in patients with nonalcoholic fatty liver disease than transient elastography. Gastroenterology 2016;150:626-37.

18 Sanyal AJ, Brunt EM, Kleiner DE, et al. Endpoints and clinical trial design for nonalcoholic steatohepatitis. Hepatology 2011;54:344-53.

19 Sanyal AJ, Friedman SL, McCullough AJ, et al. Challenges and opportunities in drug and biomarker development for nonalcoholic steatohepatitis: findings and recommendations from an American association for the study of liver Diseases-U.S. food and drug administration joint workshop. Hepatology 2015;61:1392-405.

20 Ratziu V, Harrison SA, Francque S, et al. Elafibranor, an Agonist of the Peroxisome Proliferator-Activated Receptor- $\alpha$ and $-\delta$, Induces Resolution of Nonalcoholic Steatohepatitis Without Fibrosis Worsening. Gastroenterology 2016;150:1147-59.

21 Dulai PS, Sirlin CB, Loomba R. Mri and MRE for non-invasive quantitative assessment of hepatic steatosis and fibrosis in NAFLD and NASH: clinical trials to clinical practice. $J$ Hepatol 2016;65:1006-16.

22 Costa-Silva L, Ferolla SM, Lima AS, et al. Mr elastography is effective for the non-invasive evaluation of fibrosis and necroinflammatory activity in patients with nonalcoholic fatty liver disease. Eur $J$ Radiol 2018;98:82-9.

23 Reeder SB, Robson PM, Yu H, et al. Quantification of hepatic steatosis with MRI: the effects of accurate fat spectral modeling. $J$ Magn Reson Imaging 2009;29:1332-9.

24 Konerman MA, Jones JC, Harrison SA. Pharmacotherapy for NASH: current and emerging. $J$ Hepatol 2018;68:362-75.

25 Sanyal A, Charles ED, Neuschwander-Tetri BA, et al. Pegbelfermin (BMS-986036), a PEGylated fibroblast growth factor 21 analogue, in patients with non-alcoholic steatohepatitis: a randomised, double-blind, placebo-controlled, phase $2 \mathrm{~A}$ trial. Lancet 2019;392:2705-17.

26 Harrison SA, Rinella ME, Abdelmalek MF, et al. NGM282 for treatment of non-alcoholic steatohepatitis: a multicentre, randomised, double-blind, placebo-controlled, phase 2 trial. Lancet 2018;391:1174-85. 